Departement für Nutztiere der Veterinärmedizinischen Fakultät der Universität Zürich

Klinik für Fortpflanzungsmedizin

(Direktor: Prof. Dr. W. Kähn)

Arbeit unter der Leitung von Dr. U. Eigenmann

\title{
Körperkondition und Häufigkeit von Ovarialzysten bei Braunviehkühen während der ersten $\mathbf{4 0}$ Laktationstage
}

\author{
INAUGURAL-DISSERTATION \\ zur Erlangung der Doktorwürde \\ der Veterinärmedizinischen Fakultät \\ der Universität Zürich
}

vorgelegt von

Martin Ludwig Hasler

Tierarzt

von Eschen (FL)

Genehmigt auf Antrag von

Prof. Dr. R. Thun, Referent

Prof. Dr. R. Stolla, Korreferent 


\title{
Körperkondition und Häufigkeit von Ovarialzysten bei Braunviehkühen während der ersten 40 Laktationstage
}

\author{
M.Hasler ${ }^{1}$, U. Eigenmann ${ }^{1}$, F. Janett ${ }^{2}$, R. Thun ${ }^{2}$ \\ Praxis Dr. U. Eigenmann, Degersheim, ${ }^{2}$ Klinik für Fortpflanzungsmedizin der Universität \\ Zürich
}

\section{Zusammenfassung}

Die Untersuchung wurde an 50 Milchviehkühen der Rasse Braunvieh in 21 Betrieben durchgeführt. 25 peripartal überkonditionierten Kühen (Rückenfettdicke $>38 \mathrm{~mm}$ ) wurden 25 peripartal nicht überkonditionierte Tiere (Rückenfettdicke $\leq 38 \mathrm{~mm}$ ) zugeordnet. An den Tagen 20, 30 und 40 post partum wurden die Ovarien mittels Ultraschall untersucht und 10, 15, 20, 30 und 40 Tage nach der Abkalbung die Konzentrationen von Progesteron und $17 \beta$-Oestradiol im Plasma bestimmt. Bei den peripartal überkonditionierten Tieren wurden 12 , bei den nicht überkonditionierten lediglich 1 Ovarialzyste festgestellt $(\mathrm{P}<0.05)$. Bei der ersten Untersuchung wurden alle Ovarialzysten sonografisch als Follikelthekazysten (Plasmaprogesteron $\leq 0.5 \mathrm{ng} / \mathrm{ml}$ ) beurteilt. Im weiteren Untersuchungsverlauf persistierten 3 als Thekazysten, bei 8 Zysten trat eine Luteinisierung ein und 2 Zysten heilten ab. Es gab keinerlei Hinweise, dass überkonditionierte Kühe infolge des höheren Fettansatzes vor dem Auftreten der Ovarialzysten höhere Progesteron- und/oder Östrogenwerte im Plasma aufwiesen. 



\section{Body condition and frequency of ovarian cysts in Brown Swiss cows during the first $\mathbf{4 0}$ days of lactation}

\section{Summary}

For this investigation 50 Brown Swiss cows from 21 different farms were used. Twentyfive peripartal overconditioned cows (back fat thickness $>38 \mathrm{~mm}$ ) were compared with 25 peripartal not overconditioned animals (back fat thickness $<38 \mathrm{~mm}$ ). On days 20, 30 and 40 post partum the ovaries were examined sonographically and 10,15, 20, 30 and 40 days after calving plasma concentrations of progesterone and 17- $\beta$ estradiol were determined. In peripartal overconditioned animals 12 ovarian cysts were found while only one cyst was present in not overconditioned cows $(\mathrm{P}<0.05)$. At first examination all ovarian cysts were classified by ultrasound as follicle theca cysts (progesterone $<0.5 \mathrm{ng} / \mathrm{ml}$ plasma). Follow examinations resulted in 3 cysts which persisted as theca cysts while 8 cysts became luteinized and 2 cysts completely regressed. There was no indication of increased plasma progesterone and/or estradiol concentrations in overconditioned cows with higher fat deposit before of ovarian cysts had occurred. 



\section{Einleitung}

Energiedepots in Form von Körperfett spielen im Fruchtbarkeitsgeschehen der Milchkuh eine entscheidende Rolle. Peripartal fette Tiere zeigen vermehrt Ovarialzysten und Fertilitätsprobleme (Gearhart et al., 1990; Mösenfechtel et al., 2000). Warum bei überkonditionierten Kühen zu Beginn der Laktation Ovarialzysten häufiger auftreten und um welche Zystenart es sich handelt, ist unklar. Im veterinärmedizinischen Schrifttum gibt es Hinweise, dass bei einem Energiedefizit das im Fett gespeicherte Progesteron infolge Lipolyse vermehrt freigesetzt wird und zu geringgradig erhöhten Blutkonzentrationen führen kann, die ihrerseits stille Brunst (Schopper und Claus, 1986) und die Bildung von Ovarialzysten (Noble et al., 2000; Silvia et al., 2002) auslösen können. Zudem ist aus der Humanmedizin bekannt, dass bei der Frau die Oestrogenkonzentration im Blut eng mit dem Fettanteil im Organismus korreliert ist, da die im Fettgewebe vorkommende Aromatase Androgene in Östrogene umzuwandeln vermag (Frisch, 1988; Frisch et al., 1992). Wäre dies auch bei überkonditionierten Kühen der Fall, könnten erhöhte Östrogenkonzentrationen im Blut ebenfalls ein Grund für das vermehrte Auftreten von Ovarialzysten sein (Wiltbank et al., 2002). Da noch keine hormonanalytischen Untersuchungen von überkonditionierten Kühen bereits vor dem Auftreten von Ovarialzysten vorliegen, bestand das Ziel der vorliegenden Arbeit darin, a) die Inzidenz und Art von Ovarialzysten bei peripartal über- und nicht überkonditionierten Kühen während der ersten 40 Laktationstage zu bestimmen und b) den Verlauf von Oestrogen und Progesteron vor und nach dem Auftreten von Ovarialzysten zu verfolgen.

\section{Tiere, Material und Methoden}

\section{Tiere}

Die Untersuchungen wurden an 50 klinisch gesunden Milchviehkühen der Rasse Braunvieh mit unterschiedlichem Brown-Swiss-Einkreuzungsgrad durchgeführt. Kühe mit einer Zwillingsträchtigkeit, einer Schwergeburt oder einer Retentio secundinarum wurden vom Versuch ausgeschlossen.

\section{Versuchsbetriebe und Versuchszeitraum}

Die Untersuchungen wurden in 21 auf 650 bis $1000 \mathrm{~m}$ über Meer liegenden Milchviehbetrieben im Untertoggenburg (Kanton St. Gallen) und in angrenzenden Gemeinden des Kantons Appenzell Ausserrhoden durchgeführt und dauerten von Oktober 2002 bis März 2003. Sämtliche Tiere wurden in Anbindehaltung gehalten. 


\section{Fütterung}

Die von Graswirtschaft und Rohmilchkäseproduktion geprägte Region bestimmte auch weitgehend die Art der Fütterung. Unabhängig vom Laktationsstadium erhielten die Tiere als Grundfutter Heu ad libitum und ca. $50 \mathrm{~g}$ Viehsalz. Zwei Wochen vor der Abkalbung erhielten die Kühe in zunehmenden Mengen Kraftfutter, bis sie zum Zeitpunkt des Partus 1 bis $2 \mathrm{~kg}$ eines ausgeglichenen Milchviehfutters aufnahmen. Während der ersten 3 Monate post partum bekamen die Tiere ca. $100 \mathrm{~g}$ einer Mineralsalzmischung (Calcium-Phosphor Verhältnis 2:1). $\mathrm{Ab}$ einer Milchleistung von $18 \mathrm{~kg} / \mathrm{Tag}$ wurden pro $2 \mathrm{~kg}$ Mehrleistung an Milch zusätzlich 0.3 bis $1 \mathrm{~kg}$ eines ausgeglichenen Milchviehfutters vorgelegt.

\section{Versuchsanordnung}

In Abbildung 1 ist die Versuchsanordnung mit Zeitangaben der erhobenen Parameter aufgezeichnet. Mittels Ultraschallmessung der Rückenfettdicke wurden in 21 Milchviehbetrieben 25 peripartal überkonditionierte Braunviehkühe ermittelt. Jeder überkonditionierten Kuh wurde aus dem gleichen Betrieb ein peripartal nicht überkonditioniertes Tier zugeordnet und 40 Tage nach der Abkalbung bei allen Tieren nochmals die Rückenfettdicke bestimmt. Bei sämtlichen 50 in den Versuch aufgenommenen Kühen wurden zusätzlich folgende Daten erhoben: An den Tagen 20, 30 und 40 post partum wurden Ultraschalluntersuchungen der Ovarien durchgeführt und an den Tagen 10, 15, 20, 30 und 40 nach der Abkalbung erfolgten Blutentnahmen zur Bestimmung der Progesteron- und $17 \beta-$ Oestradiol-Konzentrationen.

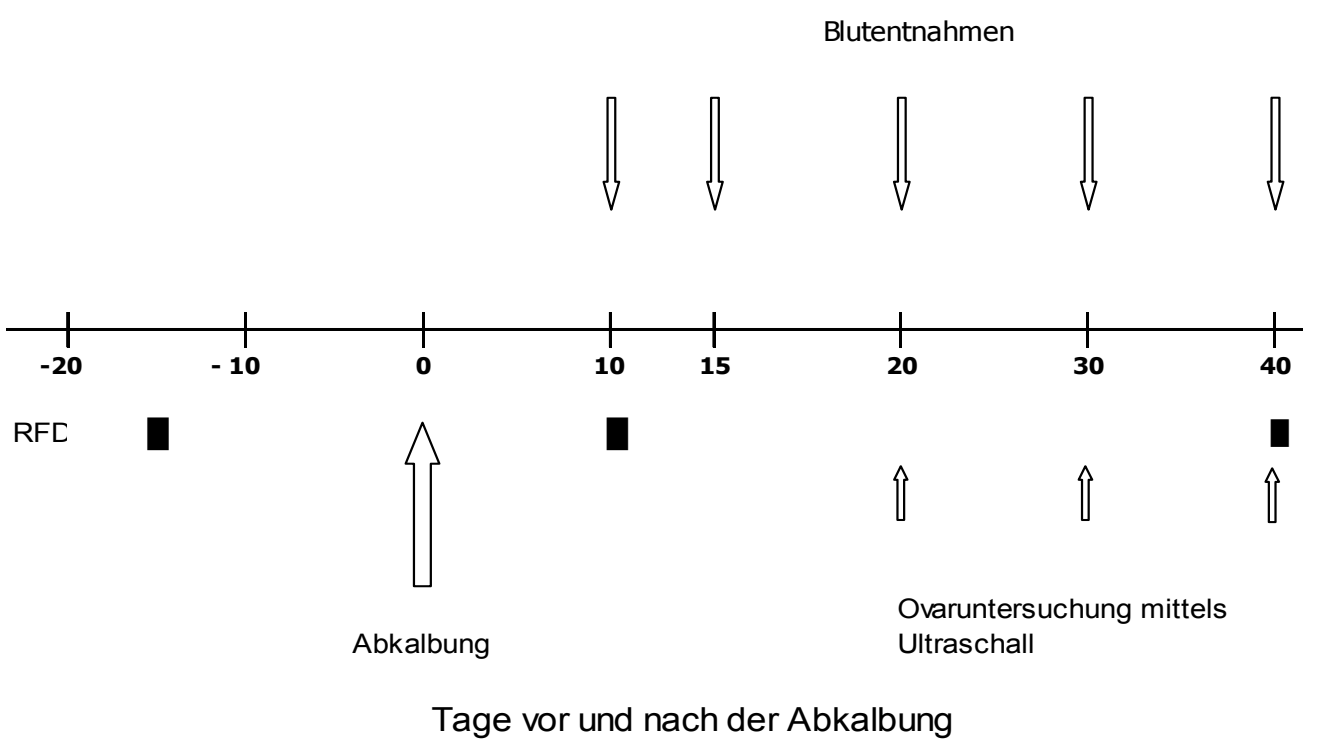

Abbildung 1: Versuchsanordnung mit Zeitangaben für die Rückenfettdickenmessung (RFD), Blutentnahmen und Ovaruntersuchung. 


\section{Milchleistung und Laktationsnummer}

Die energiekorrigierte 100-Tage-Milchleistung der peripartal über- und nicht überkonditionierten Tiere betrug im Mittel $2660 \pm 144 \mathrm{~kg}$ bzw. $2830 \pm 142 \mathrm{~kg}(\mathrm{P}>0.05)$. Von allen über- und nicht überkonditionierten Kühen waren 4 bzw. 2 Erstkalbinnen, die übrigen standen in der 2. oder einer der folgenden Laktationen.

\section{Erkrankungen}

Während der ganzen Versuchsdauer erlitten insgesamt 8 Kühe eine Allgemeinerkrankung: 5 peripartal überkonditionierte und 2 zum gleichen Zeitpunkt nicht überkonditionierte Tiere litten an Gebärparese und eine peripartal überkonditionierte Kuh entwickelte eine primäre Ketose. Sämtliche Tiere sprachen auf eine übliche Standardbehandlung an. Daneben zeigten 5 bzw. 4 peripartal über- und nicht überkonditionierte Tiere 10 Tage post partum leicht übelriechenden Vaginalausfluss. Diese Kühe wurden intrauterin mit 4 Injecur-Uterus-Stäben (1000 mg Tetracyclini hydrochloricum, $500 \mathrm{mg}$ Clioquinolum, Veterinaria AG, Zürich) behandelt.

\section{Messung der Rückenfettdicke (RFD) und Konditionsbeurteilung}

Die Rückenfettdickenmessung erfolgte mittels Ultraschall nach der Methode von Staufenbiel (1997). Sämtliche RFD-Werte wurden in mm angegeben und beinhalten neben der Fettdicke auch die Hautdicke. Die Konditionsbeurteilung sämtlicher Versuchstiere erfolgte nach den Angaben von Mösenfechtel et al. (2000) und ist in Tabelle 1 zusammengefasst. Bei jedem Tier wurde die Rückenfettdicke 10 - 20 Tage vor und 10 Tage nach dem Kalben errechnet. Der so ermittelte Durchschnittswert wurde peripartale RFD genannt. Betrug dieser Wert $>38$ oder $\leq 38 \mathrm{~mm}$ wurden die Kühe als peripartal über- bzw. nicht überkonditioniert eingestuft. Die 40 Tage post partum ermittelte RFD wurde als postpuerperale RFD bzw. Körperkondition bezeichnet.

Tabelle 1: Konditionsbeurteilung aufgrund der peripartalen und postpuerperalen Rückenfettdicke (RFD).

\begin{tabular}{ccc}
\hline peripartale RFD & postpuerperale RFD & Beurteilung \\
\hline $29-33 \mathrm{~mm}$ & $<28 \mathrm{~mm}$ & unterkonditioniert \\
$34-38 \mathrm{~mm}$ & $28-34 \mathrm{~mm}$ & optimal \\
$>38 \mathrm{~mm}$ & $>34 \mathrm{~mm}$ & überkonditioniert \\
\hline
\end{tabular}




\section{Gynäkologische Untersuchung}

Bei allen Tieren wurde am 20., 30. und 40. Laktationstag mittels Ultraschall eine Ovaruntersuchung durchgeführt. Hierzu wurde ein portables Ultraschallgerät ( Scanner 100 Vet, Pie Medical Equipment B.V., Maastricht, Niederlande) mit einem 5/7.5 MHz-LinearArray-Schallkopf verwendet. Die Diagnose „Ovarialzyste“ wurde dann gestellt, wenn auf wenigstens einem Ovar eine Blase mit einem Innendurchmesser von mehr als $25 \mathrm{~mm}$ in Grösse und Lokalisation während 10 Tagen bei gleichzeitiger Abwesenheit eines Corpus luteum persistierte. Die Unterteilung der Ovarialzysten in Follikeltheka- und FollikelluteinZysten erfolgte aufgrund der Beschaffenheit der Zystenwand und des Zystenhohlraumes: Dünnwandige echofreie Blasen wurden als Follikeltheka-, Blasen mit wenig echogebender Wand und/oder mässig echogenem Inhalt als Follikellutein-Zyste beurteilt (Farin et al., 1990). Wurde auf den Ovarien zu irgendeinem Untersuchungszeitpunkt ein Corpus luteum festgestellt, wurde die Kuh als zyklisch bewertet (Wiltbank et al., 2002). Ging dem Corpus luteum eine Ovarialzyste voraus, wurde diese als abgeheilt beurteilt (Silvia et al., 2002). Waren zu allen Untersuchungszeitpunkten nur Follikel mit Innendurchmesser $<20 \mathrm{~mm}$ und kein C. luteum sichtbar, galt das Versuchstier als anovulatorisch (Lucy et al., 1992; Wiltbank et al., 2002).

\section{Hormonbestimmung}

Die Blutentnahme erfolgte aus der Vena epigastrica caudalis in heparinisierte Blutröhrchen. Die Blutproben wurden anschliessend bei $3000 \mathrm{U} / \mathrm{min}$ während 5 Minuten zentrifugiert und das gewonnene Plasma bei $-25{ }^{\circ} \mathrm{C}$ gefroren. Die Bestimmung von Progesteron (Progesterone \# 246504 bovine Radioimmunoassay kit, Immunotech, Marseille) und Östradiol-17 $\beta$ (Estradiol, \# 2464 bovine Radioimmunoassay kit, Immunotech, Marseille) erfolgte am Institut für Physiologie, Technische Universität München. Die Empfindlichkeit für Progesteron und Östradiol-17ß betrug $0.1 \mathrm{ng} / \mathrm{ml}$ (Prakash et al., 1987) bzw. 0.8 pg/ml (Meyer et al., 1990).

\section{Statistische Auswertung}

Die statistische Auswertung erfolgte nach Weber (1995). In sämtlichen Abbildungen wurden Mittelwert und Standardfehler dargestellt, bei Häufigkeitsverteilungen gelangte die absolute Anzahl zur Auswertung. Bei Mittelwertvergleichen nicht normalverteilter Stichproben wurde der U-Test nach Mann und Whitney durchgeführt. Häufigkeitsverteilungen wurden mit der Zwei-mal-k-Tafel auf signifikante Unterschiede geprüft. Zwischen der peripartalen und postpuerperalen RFD wurde der Korrelationskoeffizient berechnet. Den statistischen Auswertungen wurde eine Irrtumswahrscheinlichkeit von $\mathrm{P}<0.05$ zugrunde gelegt. 


\section{Ergebnisse}

\section{Beziehung zwischen peripartaler und postpuerperaler RFD}

Der Korrelationskoeffizient zwischen der Rückenfettdicke beim Abkalben und derjenigen 40 Tage p.p. war signifikant und betrug 0.88 . Von den 25 peripartal überkonditionierten Tieren waren 40 Tage nach der Abkalbung immer noch 18 überkonditioniert (Abb. 2) und nur 7 Kühe optimal konditioniert. Die peripartal nicht überkonditionierte Gruppe $(n=25)$ setzte sich aus 9 optimal und 16 unterkonditionierten Kühen zusammen. Von den 9 optimal konditionierten Tieren wiesen 8 auch postpuerperal eine optimale Körperkondition auf und lediglich 1 Kuh war postpuerperal unterkonditioniert. Von den 16 peripartal unterkonditionierten Tieren wiesen 13 auch postpuerperal einen zu geringen Fettansatz auf und nur 3 Tiere schafften den Sprung in den postpuerperal optimal konditionierten Zustand.

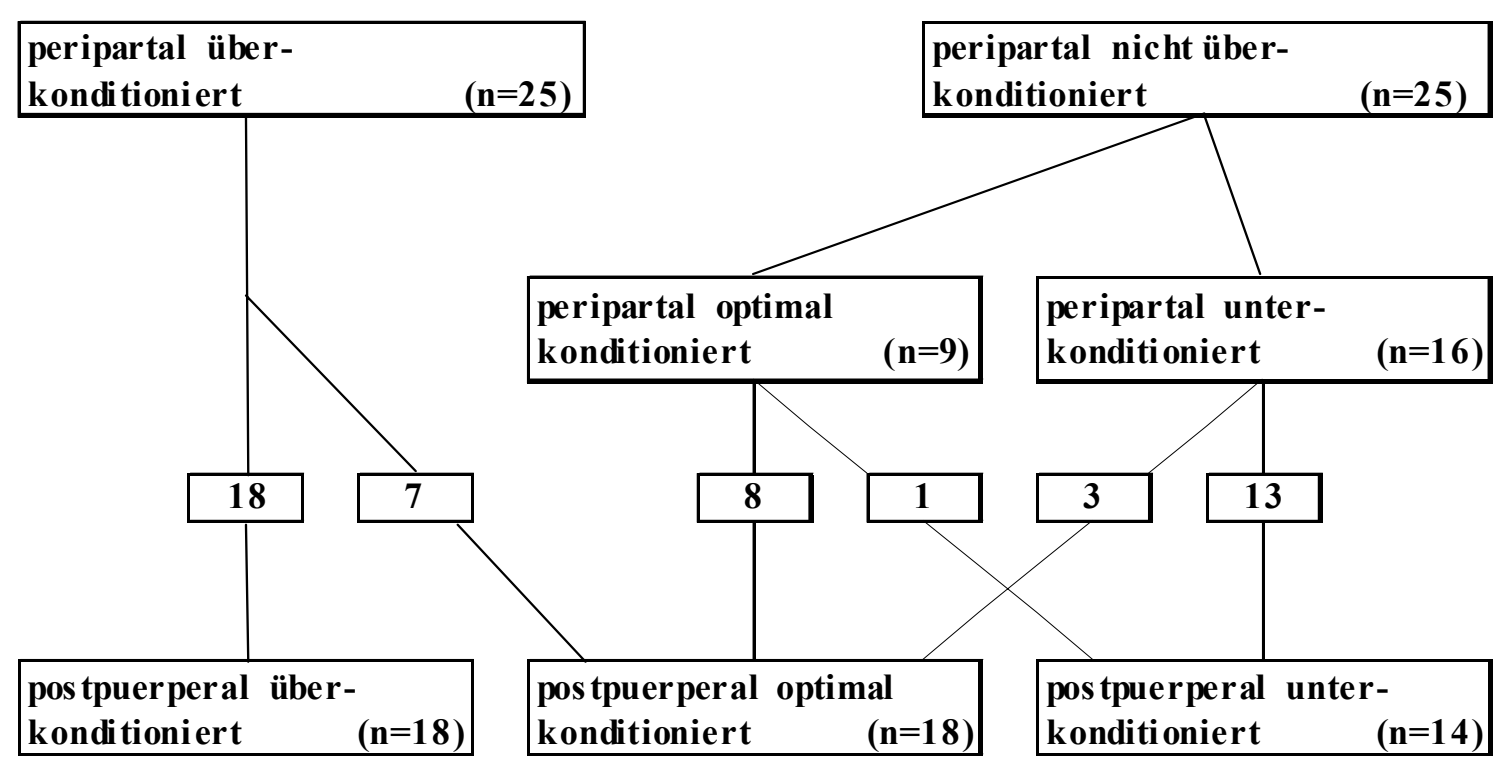

Abbildung 2: Peripartale und postpuerperale (40 Tage post partum) Körperkondition bei den Versuchstieren.

\section{Vorkommen und Charakterisierung von Ovarialzysten}

Die peripartal überkonditionierte Gruppe wies während der ersten 40 Laktationstage 12 und damit signifikant mehr Ovarialzysten auf als die zum gleichen Zeitpunkt nicht überkonditionierten Tiere mit lediglich einer Ovarialzyste (Tab. 2). 
Tabelle 2: Häufigkeit und Schicksal von Ovarialzysten während der ersten 40 Laktationstage.

\begin{tabular}{lccc}
\hline $\begin{array}{l}\text { Versuchstiere } \\
(\mathbf{n}=\mathbf{5 0})\end{array}$ & total & Persistenz & Rückbildung \\
\hline $\begin{array}{l}\text { peripartal } \\
\text { überkonditioniert } \\
(\mathrm{n}=25)\end{array}$ & $12^{\mathrm{a}}$ & $10^{\mathrm{a}}$ & 2 \\
$\begin{array}{l}\text { peripartal nicht } \\
\text { überkonditioniert } \\
(\mathrm{n}=25)\end{array}$ & $1^{\mathrm{b}}$ & $1^{\mathrm{b}}$ & 0 \\
\hline
\end{tabular}

${ }^{\mathrm{a}, \mathrm{b}}$ Werte mit unterschiedlichen Indices sind signifikant verschieden $(\mathrm{P}<0.05)$

Elf der 13 Ovarialzysten wurden erstmals 20 Tage p.p., die übrigen 2 Zysten erst 10 Tage später beobachtet. Bei der ersten Untersuchung konnten alle Ovarialzysten sonografisch als Follikelthekazysten eingestuft werden. Im weiteren Verlauf blieben von den insgesamt 13 Thekazysten 3 bis zum 40. Tag p.p. weiterhin als Follikelthekazysten bestehen (Plasmaprogesteronwerte $\leq 0.5 \mathrm{ng} / \mathrm{ml}$ ). Bei 2 Tieren mit Follikelthekazysten hatte der Zystendurchmesser bis zum 40. Tag p.p. abgenommen, während sich auf dem anderen Ovar ein Corpus luteum (Plasmaprogesteron 2.5 und $4.3 \mathrm{ng} / \mathrm{ml}$ ) gebildet hatte und die Zyste somit als funktionell abgeheilt betrachtet wurde. Bei den restlichen 8 Tieren mit Follikelthekazysten konnte spätestens am 40. Tag p.p. eine Luteinisierung der Zyste (mässig echogene Wand und/oder echogener Inhalt im Zystenlumen) beobachtet werden. Die Plasmaprogesteronwerte schwankten zwischen 0.6 und $10.2 \mathrm{ng} / \mathrm{ml}$ mit einem Durchschnittswert von $2.9 \mathrm{ng} / \mathrm{ml}$ am 40 . Tag p.p. In Abbildung 3 ist die Umwandlung einer Follikelthekazyste in eine Follikelluteinzyste dargestellt. 

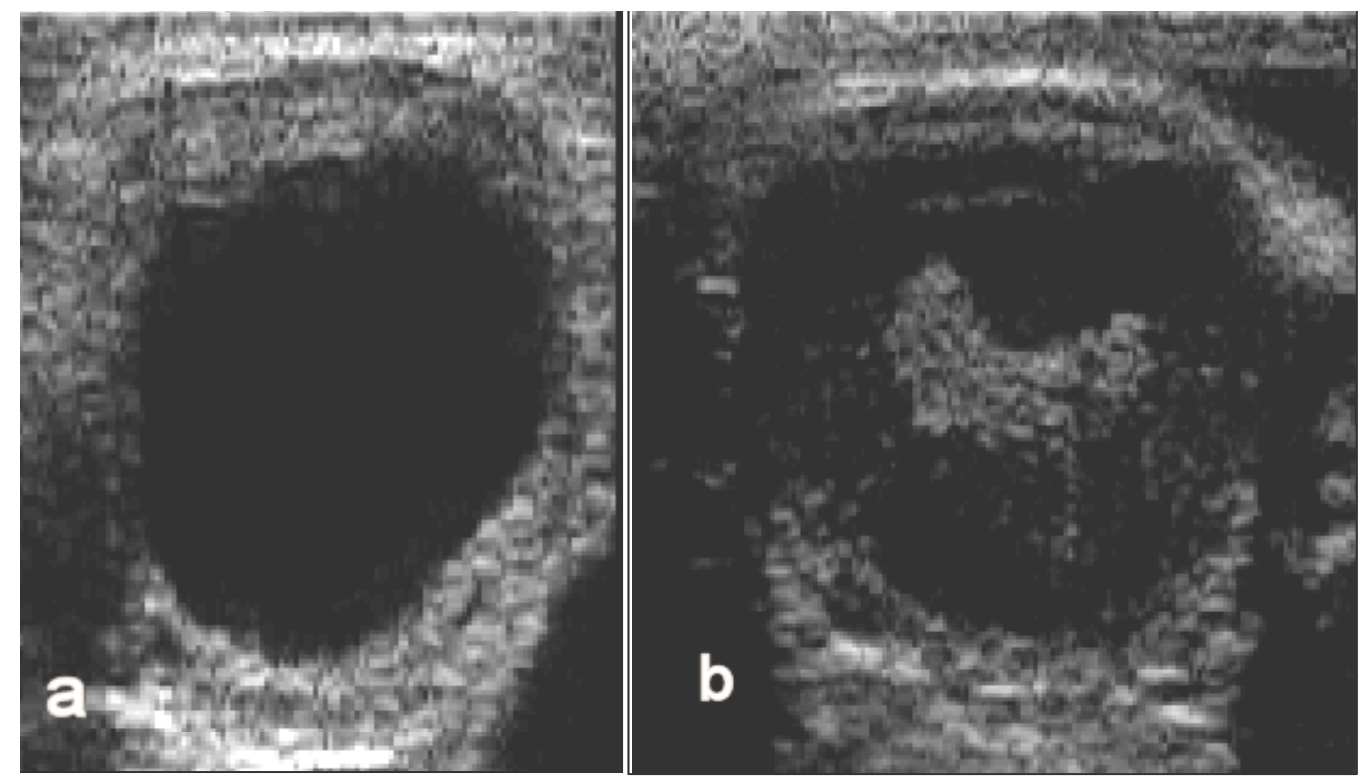

Abbildung 3: Rechtes Ovar mit a) Follikelthekazyste ( $\varnothing 35 \mathrm{~mm}) 30$ Tage p.p.und b) Luteinzyste $(\varnothing 33 \mathrm{~mm}) 10$ Tage später.

\section{Einsetzen des Ovarzyklus}

Bei den peripartal über- und nicht überkonditionierten Tieren setzte während der ersten 40 Laktationstage bei insgesamt 14 bzw. 11 Tieren ein Zyklus ein (Tab. 3), was auch sonografisch (deutlich sichtbares Corpus luteum) erhärtet werden konnte.

Tabelle 3: Einsetzen des Ovarzyklus während der ersten 40 Laktationstage. Zwischen überkonditionierten und nicht überkonditionierten Tieren bestehen keine signifikanten $(\mathrm{P}<0.05)$ Unterschiede.

\begin{tabular}{lccc}
\hline $\begin{array}{l}\text { Versuchstiere } \\
(\mathbf{n}=\mathbf{5 0})\end{array}$ & total & $\begin{array}{c}\text { Einsetzen des Ovarzyklus } \\
\text { ohne vorausgegangene } \\
\text { Zyklusstörung }\end{array}$ & mit Ovarialzyste \\
\hline $\begin{array}{l}\text { peripartal } \\
\text { überkonditioniert } \\
(\mathrm{n}=25)\end{array}$ & 14 & 12 & 2 \\
$\begin{array}{l}\text { peripartal nicht } \\
\text { überkonditioniert } \\
(\mathrm{n}=25)\end{array}$ & 11 & 11 & 0 \\
\hline
\end{tabular}


Die Plasmaprogesteronwerte der Tiere mit deutlich erkennbarem Corpus luteum schwankten zwischen 1.1 bis $15.3 \mathrm{ng} / \mathrm{ml}$. Bei 2 peripartal überkonditionierten Kühen ging dem Einsetzen des Ovarzyklus eine Follikelthekazyste voran. Elf bzw. 14 über- und nicht überkonditionierte Tiere (Tab. 4) blieben während der ersten 40 Laktationstage azyklisch. Bei den peripartal überkonditionierten Kühen ohne Zyklus lag in 10 Fällen eine persistierende Ovarialzyste und in einem Fall eine Azyklie, gekennzeichnet durch anovulatorische Follikel, vor. Bei den 14 peripartal nicht überkonditionierten Kühen ohne Zyklus während der ersten 40 Laktationstage lag in einem Fall eine persistierende Ovarialzyste vor, in allen anderen Fällen waren zu den Untersuchungszeitpunkten 20, 30 und 40 Tage p.p. auf mindestens einem Ovar immer anovulatorische Follikel (Durchmesser 12-18 mm) vorhanden; Corpora lutea fehlten und die Plasmaprogesteronwerte lagen unterhalb der Nachweisgrenze. In Tabelle 4 wurden die peripartal nicht überkonditionierten Tiere in eine optimal $(n=9)$ und eine unterkonditionierte Gruppe $(\mathrm{n}=16)$ unterteilt: Bei den 9 optimal konditionierten Tieren lag in 2 Fällen eine Azyklie (anovulatorische Follikel) vor, während dies bei den 16 unterkonditionierten Kühen 11 mal auftrat $(\mathrm{P}<0.05)$. Zusätzlich lag bei einer peripartal unterkonditionierten Kuh eine durch eine Ovarialzyste bedingte Azyklie vor.

Tabelle 4: Nichteinsetzen des Ovarzyklus während der ersten 40 Laktationstage.

\begin{tabular}{lccc}
\hline $\begin{array}{l}\text { Versuchstiere } \\
(\mathbf{n}=\mathbf{5 0})\end{array}$ & total & $\begin{array}{c}\text { Nichteinsetzen des Ovarzyklus (Azyklie) } \\
\text { persistierende } \\
\text { Ovarialzyste }\end{array}$ & $\begin{array}{c}\text { anovulatorische } \\
\text { Follikel }\end{array}$ \\
\hline $\begin{array}{l}\text { peripartal } \\
\text { überkonditioniert } \\
(\mathrm{n}=25)\end{array}$ & 11 & $10^{\mathrm{a}}$ & $1^{\mathrm{a}}$ \\
$\begin{array}{l}\text { peripartal nicht } \\
\text { uberkonditioniert } \\
(\mathrm{n}=25) \\
\begin{array}{l}\text { optimal konditioniert } \\
(\mathrm{n}=9)\end{array}\end{array}$ & 14 & $2^{\mathrm{b}}$ & $13^{\mathrm{b}}$ \\
$\begin{array}{l}\text { unterkonditioniert } \\
(\mathrm{n}=16)\end{array}$ & $12^{\mathrm{y}}$ & 0 & $2^{\mathrm{x}}$ \\
\hline
\end{tabular}

${ }^{\mathrm{a}, \mathrm{b}}$ Werte mit unterschiedlichen Indizes sind signifikant verschieden $(\mathrm{P}<0.05)$

${ }^{\mathrm{x}, \mathrm{y}}$ Werte mit unterschiedlichen Indizes sind signifikant verschieden $(\mathrm{P}<0.05)$ 


\section{Hormonverlauf}

In den Abbildungen 4 und 5 sind die Oestradiol- und Progesteron-Konzentrationen im Plasma bei den peripartal überkonditionierten Kühen mit und ohne Ovarialzysten sowie bei den nicht überkonditionierten Tieren ohne Ovarialzysten dargestellt. Da nur ein Tier der peripartal nicht überkonditionierten Gruppe eine Ovarialzyste aufwies, wurden die Hormonwerte dieses Tieres in den Abbildungen 4 und 5 nicht berücksichtigt. Die mittleren PlasmaOestradiolkonzentrationen (Abb. 4) waren bei den überkonditionierten Kühen mit Ovarialzysten 15 und 20 Tage p.p. signifikant höher (10.0 bzw. $12.2 \mathrm{pg} / \mathrm{ml})$ als bei den überkonditionierten Kühen ohne Zysten (4.7 bzw. $4.1 \mathrm{pg} / \mathrm{ml})$ sowie den nicht überkonditionierten Tieren ohne Zysten (3.6 bzw. 4.2 pg/ml).

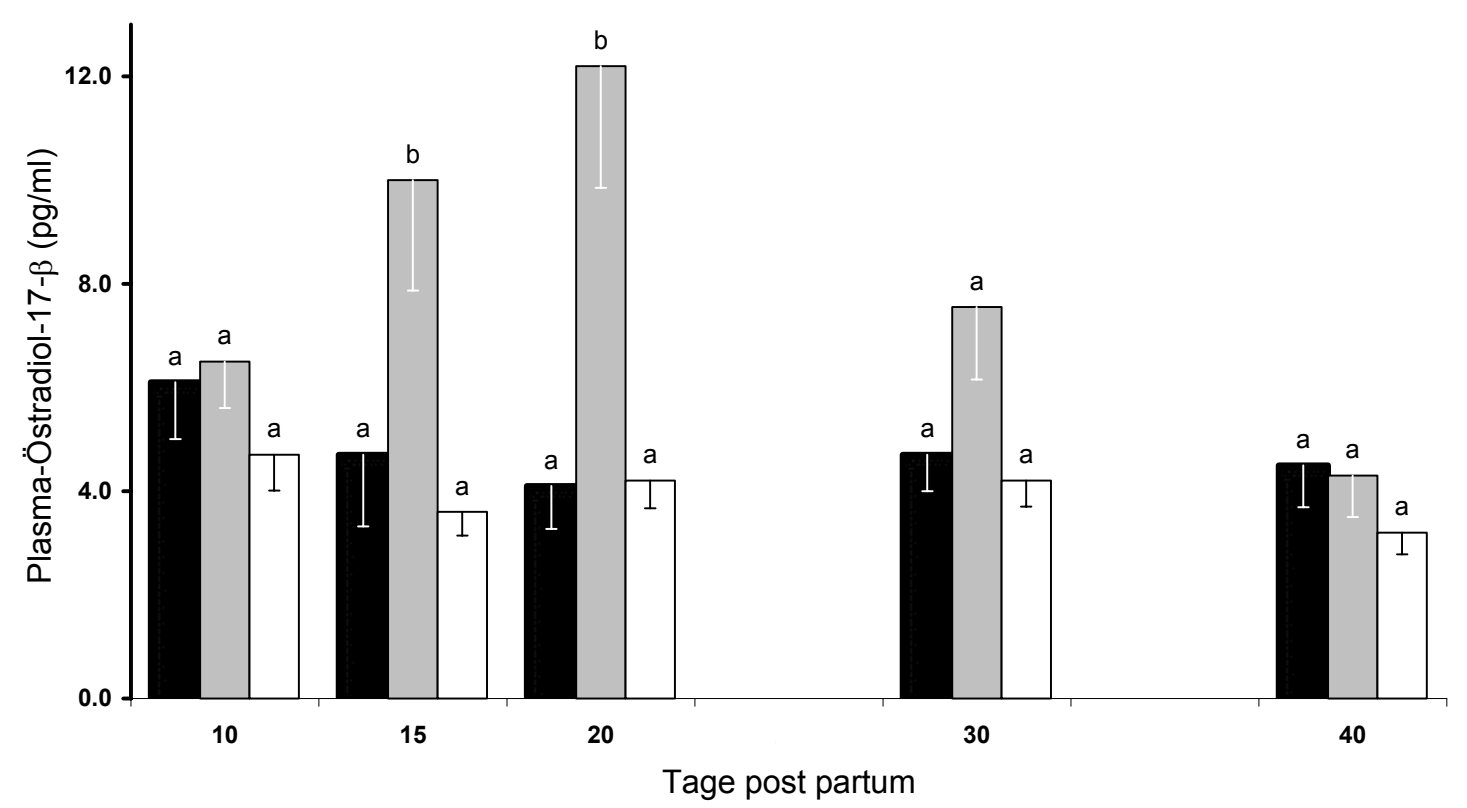

Abbildung 4: Östradiol-17 $\beta$-Konzentrationen im Plasma bei überkonditionierten Kühen ohne $(\square \mathrm{n}=13)$ und mit $(\square \mathrm{n}=12)$ Ovarialzysten sowie bei nicht überkonditionierten Kühen ohne ( $\square \mathrm{n}=24)$ Ovarialzysten nach der Abkalbung.

${ }^{\mathrm{a}, \mathrm{b}}$ Werte mit unterschiedlichen Indizes unterscheiden sich signifikant $(\mathrm{P}<0.05)$

Die durchschnittlichen Progesteronkonzentrationen im Blut (Abb. 5) waren bei den überkonditionierten Tieren ohne Zysten an sämtlichen Messzeitpunkten höher als bei den überkonditionierten Kühen mit Ovarialzysten und den nicht überkonditionierten Kühen ohne Ovarialzysten. Die Mittelwerte der 3 Gruppen waren jedoch zu keinem Zeitpunkt signifikant verschieden. 


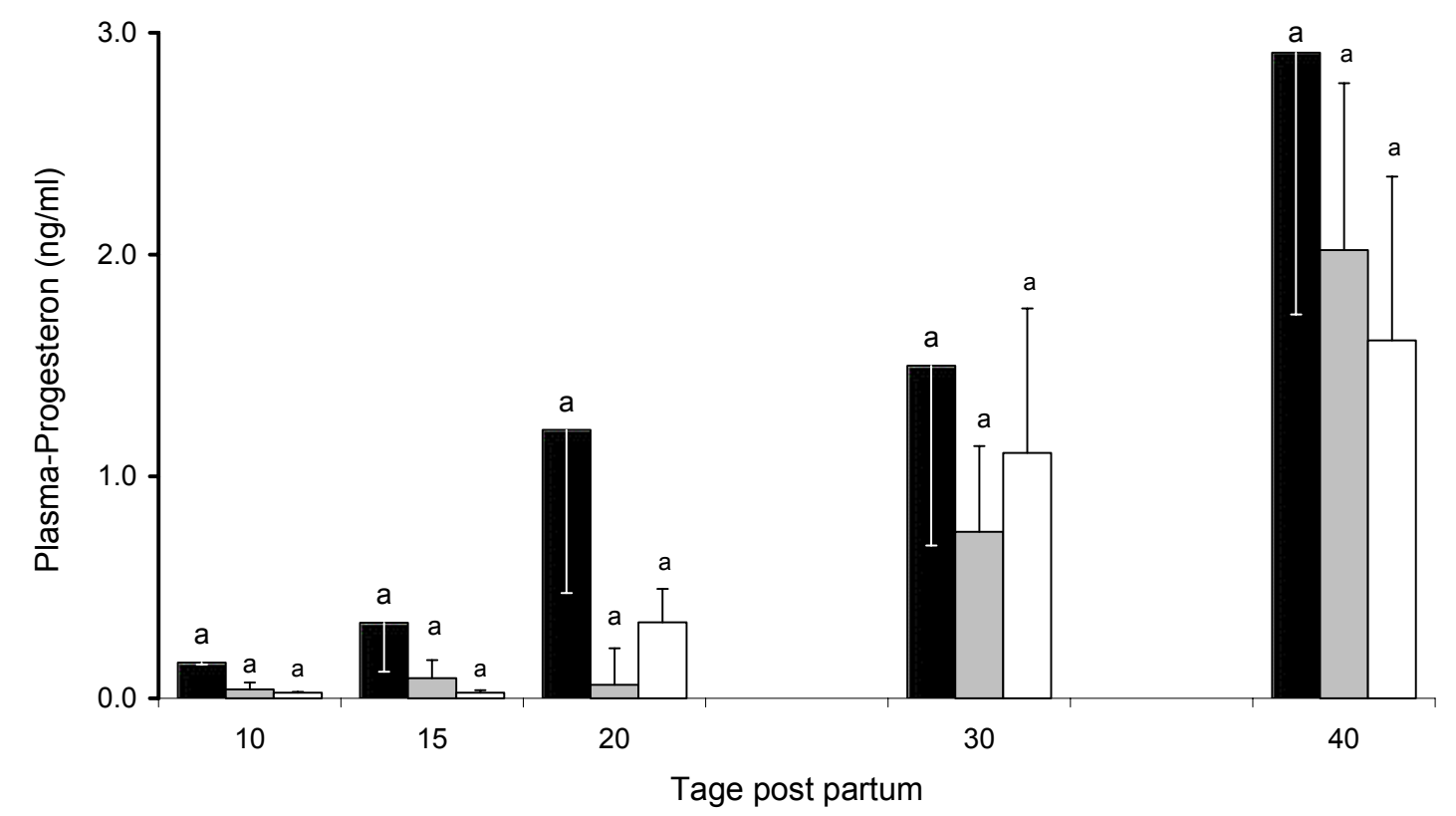

Abbildung 5: Progesteronkonzentrationen im Plasma bei überkonditionierten Kühen ohne $(\mathbf{n}=13)$ und mit $(\square \mathrm{n}=12)$ Ovarialzysten sowie bei nicht überkonditionierten Kühen ohne $(\square \mathrm{n}=24)$ Ovarialzysten nach der Abkalbung.

${ }^{\mathrm{a}, \mathrm{b}}$ Werte mit unterschiedlichen Indizes unterscheiden sich signifikant $(\mathrm{P}<0.05)$

\section{Diskussion}

Die Ergebnisse unserer Untersuchungen haben deutlich gezeigt, dass die peripartale Körperkondition einen bedeutenden Einfluss auf die Kondition 6 Wochen nach der Abkalbung hat. Diesen Zusammenhang haben auch Mösenfechtel et al. (2000) beschrieben, die zwischen der peripartalen und postpuerperalen Rückenfettdicke eine grosse Abhängigkeit (Korrelationskoeffizient 0.8) nachweisen konnten. Dies bedeutet, dass es für eine Kuh schwierig ist, während der Rastzeit die bei der Abkalbung vorhandene Körperkondition zu verbessern. Daraus kann gefolgert werden, dass die optimale Körperkondition bereits zum Abkalbezeitpunkt vorhanden sein muss, damit in der Startphase bei leistungsbezogener Fütterung der Ovarzyklus in Gang kommt. Weiter haben unsere Untersuchungen deutlich erkennen lassen, dass peripartal überkonditionierte Kühe wesentlich häufiger an Ovarialzysten erkranken als peripartal nicht überkonditionierte Tiere. Diese Beobachtung stellten auch Gearhart et al. (1990), Morrow et al. (1997) sowie Mösenfechtel et al. (2000) fest. Bei der ersten Untersuchung wurden alle Ovarialzysten sowohl sonografisch als auch aufgrund der tiefen Plasmaprogesteronwerte als Follikelthekazysten eingestuft. Aufgrund der sonografischen Untersuchungen kann davon ausgegangen werden, dass die zwischen 20 und 30 Tagen p.p. beobachteten Ovarialzysten anfänglich Follikelthekazysten sind. Bislang gibt es 
unseres Wissens keine Untersuchungen, die zu diesem Zeitpunkt nur Follikelthekazysten nachweisen konnten. Allerdings vermuten auch Garverick (1997) sowie Lopez-Gatius und Lopez-Bejar (2002), dass Luteinzysten ursprünglich Follikelthekazysten waren. Dies würde auch erklären, warum in der Literatur das Verhältnis von Follikeltheka- zu Follikelluteinzysten sehr unterschiedlich angegeben wird. So waren im Patientengut von Leidl et al. (1979) weniger als 10\% der Ovarialzysten Luteinzysten, während im Untersuchungsgut von Farin et al. (1990) und Ribadu et al. (1994) über 60\% der Zysten luteinisiert waren. Die bei unseren Versuchstieren ursprünglich vorhandenen Thekazysten luteinisierten, persistierten oder bildeten sich durch Ingangkommen eines Ovarialzyklus mit anschliessender Lutealphase zurück. Dass Follikelthekazysten während längerer Zeit persistieren und so das Zyklusgeschehen blockieren können, wurde schon früher beschrieben (Garverick, 1997). Auch die Rückbildung von Ovarialzysten durch Ovulation eines heranreifenden parazyklischen Tertiärfollikels ist in erst kürzlich erschienenen Arbeiten (Grunert, 1999; Lopez-Gatius et al., 2002; Wiltbank et al., 2002) dokumentiert. Dabei kann die „Selbstheilungsrate“ von Eierstockszysten ganz unterschiedlich ausfallen. In Untersuchungen von Lopez-Gatius et al. (2002) mit über 100 an Zysten erkrankten Kühen bildeten sich die Ovarialzysten bei knapp $40 \%$ der Tiere ohne Behandlung zurück. Eine zunehmende Luteinisierung der Follikelthekazysten hingegen, wie wir dies bei 8 von 13 Zysten beobachtet haben, wird in der Literatur (Kesler et al., 1980; Mujuni et al., 1993) nur selten erwähnt. Die Luteinzellen der Zysten lassen sich licht- und elektronenmikroskopisch nicht von denen periodischer Gelbkörper unterscheiden ( Gasse et al., 1984; Peukert-Adam et al., 1983 ) und die Progesteronsekretion von Luteinzysten entspricht bei ausgeprägter Luteinisierung derjenigen von Corpora lutea periodica ( Günzler et al., 1977; Leidl et al., 1979; Jeffcoate und Ayliffe, 1995). Aus diesem Grunde betrachten Gasse et al. (1984) sowie Grunert und Zerbe (1997) Luteinzysten als Ausdruck einer vorübergehenden Ovulationsstörung, nicht aber als ein das Zyklusgeschehen des Tieres nachhaltig beeinträchtigendes Gebilde. Träfe diese Aussage zu, würde in unserem Untersuchungsgut ein grosser Teil der Follikelthekazysten (8 von 13) durch die Umwandlung in eine Luteinzyste zur Abheilung gebracht. Ob diese „Selbstheilung“ allerdings auch bei Zysten mit partieller Luteinisierung und niedrigen Progesteronwerten im Blut eintritt, darf bezweifelt werden (Grunert, 1999). So stellten Silvia et al. (2002) bei „Zystentieren“ mit tiefen Blut-Progesteronwerten von 0.1 bis $0.9 \mathrm{ng} / \mathrm{ml}$ fest, dass die bestehenden Ovarialzysten in 76\% der Fälle durch neue Zysten ersetzt wurden. Daher wäre es sinnvoll, in weiteren Arbeiten das Schicksal von Luteinzysten ohne jegliche Behandlungsmassnahme über einen längeren Zeitraum zu verfolgen. 
Während der ersten 40 Laktationstage setzte der Ovarzyklus bei den über- und nicht überkonditionierten Kühen etwa gleich häufig ein. Der Grund für das Nichteinsetzen des Zyklus waren bei den peripartal überkonditionierten Kühen hauptsächlich persistierende Ovarialzysten, bei den peripartal nicht überkonditionierten Tieren lag die Ursache vorwiegend in einem Ausbleiben der Ovulation dominanter Follikel. Derartige anovulatorische Zustände sind bei Kühen nach der Abkalbung verschiedentlich beschrieben (Lucy et al., 1992; Wiltbank et al., 2002). In unserer Untersuchung waren derart azyklische Kühe vor allem solche, die bereits peripartal unterkonditioniert waren. Dies weist darauf hin, dass eine Kuh für das Ingangkommen einer normalen Zyklusaktivität nach dem Partus eine minimale Körperkondition, d.h. einen minimalen Körperfettanteil braucht (Bishop et al., 1994; Bossis et al., 2000; Imakawa et al.,1986; Mösenfechtel et al., 2000; Richards et al., 1989). Zum Beispiel setzte der Ovarzyklus bei Mutterkühen mit schlechter Körperkondition bei der Abkalbung später ein als bei Kühen mit mässiger Kondition (Bishop et al., 1994). Moreira at al. (2000) konnten zeigen, dass ein Synchronisationsprogramm bei Tieren mit einem Körperkonditionsindex von $<2.5$ (5-Punkte-Skala) weit weniger Erfolg hatte, als bei Tieren mit einem höheren Index. In der Arbeit von Mösenfechtel et al. (2000) zeigten 56\% der postpuerperal unterkonditionierten Kühe bis zum 65. Tag nach der Abkalbung keinen Oestrus, während postpuerperal optimal konditionierte Tiere in nur 19\% der Fälle anöstrisch blieben. Nach Studer (1998) sind bei Kühen mit einem Körperkonditionsindex von 2 und weniger 60 Tage post partum während langen Perioden keine Funktionsstadien auf den Ovarien vorhanden. Dieses Ergebnis zeigt, dass bei Unterschreiten eines bestimmten Körperfettanteils die Zyklusaktivität sistieren kann. Ähnliche Verhältnisse kennen wir auch aus der Humanmedizin, wo insbesondere Langstreckenläuferinnen amenorrhoeisch werden, sobald ein Mindestanteil an Fettgewebe unterschritten wird (Frisch et al., 1992).

Die hormonanalytischen Untersuchungen liefern keinerlei Hinweise, dass überkonditionierte Kühe als Folge eines vermehrten Fettabbaus vor der Zystenbildung erhöhte Progesteronkonzentrationen im Plasma aufweisen. Die leicht erhöhten Progesteronwerte der überkonditionierten Kühe ohne Ovarialzysten sind auf die Anwesenheit eines Gelbkörpers bei 9 Tieren am 20. Tag p.p. und bei 8 Tieren am 30. Tag p.p. zurückzuführen. Damit scheint die Hypothese von Schopper und Claus (1986), Progesteron würde bei fetten Kühen infolge einer gesteigerten Lipolyse in grösseren Mengen freigesetzt und sei möglicherweise Ursache für gehäuft auftretende stille Brunsten, unwahrscheinlich. Auch die Östrogenwerte der über- und nicht überkonditionierten Kühe ohne Zysten sind ähnlich, was bei der fetten Kuh mit grosser Wahrscheinlichkeit auf einen anderen Mechanismus schliessen lässt als bei der obesen Frau, 
bei der die im peripheren Fettgewebe aus Androgenen produzierten Oestrogene mehr als ein Drittel der gesamten Oestrogenproduktion ausmachen (Frisch, 1988; Frisch et al., 1992). Daraus kann gefolgert werden, dass die bei den überkonditionierten Kühen mit Zysten wesentlich erhöhten Östrogenmengen im Blut von Follikelthekazysten mit noch intakter Granulosazellschicht stammen (Silvia et al., 2002). Die Ursache von vermehrt auftretenden Ovarialzysten bei überkonditionierten Kühen bleibt weiterhin unbekannt. Inwieweit das im Fettgewebe produzierte Leptin, das die ovarielle Steroidsynthese hemmen soll (Barb et al., 1999), für die Entstehung von Ovarialzysten eine Rolle spielt, bleibt weiterhin abzuklären.

Die vorliegenden Untersuchungen haben gezeigt, dass die Eierstocksfunktion bei der puerperalen Kuh wesentlich von der Körperkondition, d.h. vom Körperfettanteil abhängig ist. Ist der Fettanteil zu hoch oder zu niedrig, treten Störungen der Ovarfunktion in Form von Follikelthekazysten bzw. anovulatorischen Zyklen auf.

\section{Dank}

Für die Bestimmungen von Progesteron und Oestradiol möchten wir Prof. Dr. D. Schams, Institut für Physiologie, Technische Universität München, Freising-Weihenstephan, bestens danken. 


\section{Literatur}

Barb C.R., Barrett J.B., Kraeling R.R., Rampacek G.B.: Role of leptin in modulating neuroendocrine function: A metabolic link between the brain-pituitary and adipose tissue. Reprod. Dom. Anim. 1999, 34, 112-125.

Bishop D.K., Wettemann R.P., Spicer L.J.: Body energy reserves influence the onset of luteal activity after early weaning of beef cows. J. Anim. Sci. 1994, 72: 2703-2708.

Bossis I., Wettemann R.P., Welty S.D., Vizcarra J., Spicer L.J.: Nutritionally induced anovulation in beef heifers: Ovarian and endocrine function during realimentation and resumption of ovulation. Biol. Reprod. 2000, 62: 1436-1444.

Farin P.W., Youngquist R.S., Parfet J.R., Garverick H.A.: Diagnosis of luteal and follicular ovarian cysts in dairy cows by sector scan ultrasonography. Theriogenology 1990, 34: 633642.

Frisch R.E.: Fatness und Fertility. Sci. Am. 1988, 258: 88-95.

Frisch R.E., Snow R.C., Johnson L.A., Gerard B., Barbieri R., Rosen B.: Magnetic resonance imaging of overall and regional body fat, estrogen metabolism, and ovulation of athletes compared to controls. J. Clin. Endocrinol. Metab. 1992, 77: 471-477.

Garverick H.A.: Ovarian follicular cysts in dairy cows. J Dairy Sci 1997, 80: 995-1004.

Gearhart M.A., Curtis C.R., Erb H.N., Smith R.D., Sniffen C.J., Chase L.E., Cooper M.D.: Relationship of changes in condition score to cow health in holsteins. J. Dairy Sci. 1990, 73: 3132-3140.

Gasse H., Peukert -Adam I., Schwarz R., Grunert E.: Die Stellung der Follikel-Lutein-Zyste im Zyklusgeschehen des Rindes: Histologische, zytologische und hormonanalytische Untersuchungen. Zbl. Vet. Med. A 1984, 31: 548-556.

Grunert E.: Ovarielle Dysfunktionen. In: Fertilitätsstörungen beim weiblichen Rind. Hrsg. Grunert E. und Berchtold M., Paul Parey Buchverlag, Berlin, 1999, 111-135.

Grunert E., Zerbe H.: Diagnose und Therapie der Follikel-Lutein-Zysten des Rindes. Prakt. Tierarzt, coll. vet. 1997, XXVII: 92-96.

Günzler O., Fastner S., Huber E.: Versuche zur Beeinflussung sog. Luteinzysten beim Rind mit dem Prostaglandinanalog „ESTRUMATE“. Tierärztl. Umschau 1977, 9: 466-472. 
Imakawa K., Day M.L., Garcia-Winder M., Zalesky D.D., Kittok R.J., Schanbacher B.D., Kinder J.E.: Endocrine changes during restoration of estrous cycles following induction of anestrus by restricted nutrient intake in beef heifers. J. Anim. Sci. 1986, 63: 565-571.

Jeffcoate I.A., Ayliffe T.R.: An ultrasonographic study of bovine cystic ovarian disease and is treatment. Vet. Rec. 1995, 136: 406-410.

Kesler D. J., Garverick H. A., Caudle A. B., Elmore R. G., Youngquist R. S., Bierschwal C. J.: Reproductive hormone and ovarian changes in cows with ovarian cysts. J. Dairy Sci. 1980, 63: 166-170.

Lopez-Gatius F., Santolaria P., Yaniz J., Fenech M., Lopez-Bejar M.: Risk factors for postpartum ovarian cysts and their spontaneous recovery or persistence in lactating dairy cows. Theriogenology 2002, 58: 1623-1632.

Lopez-Gatius F., Lopez-Bejar M.: Reproductive performance of dairy cows with ovarian cysts after different GnRH and cloprostenol treatments. Theriogenology 2002, 58: 13371348.

Leidl W., Stolla R., Hundschell C., Bostedt H.: Zur Ovarialzyste des Rindes. I. Klassifizierung und Diagnose. Berl. Münch. Tierärztl. Wochenschr. 1979, 92: 369-376.

Lucy M.C., Savio J.D., Badinga L., De La Sota R.L., Thatcher W.W.: Factors that affect ovarian follicular dynamics in cattle. J.Anim.Sci. 1992, 70: 3615-3626.

Meyer H. H. D., Sauerwein H., Mutayoba B. M.: Immunoaffinity chromatography and a biotin-streptavidin amplified enzymeimmunoassay for sensitive and specific estimation of estradiol-17- $\beta$. J. Steroid. Biochem. 1990, 35: 263-269.

Moreira F., Risco C., Pires M.F.A., Ambrose J.D., Drost M., DeLorenzo M., Thatcher W.W.: Effect of body condition on reproductive effency of lacting dairy cows receiving a timed insemination. Theriogenology 2000, 53:1305-1319.

Morrow D.A., Hillman D., Dade A.W., Kitchen Y.: Clinical investigations of dairy herd with fat cow-syndrome. J. Am. Vet. Med. Assoc. 1997, 174:161-167.

Mösenfechtel St., Eigenmann U.J., Rüsch P., Wanner M.: Rückenfettdicke und Fruchtbarkeit bei Braunviehkühen. Schweiz. Arch. Tierheilk. 2000, 142: 679-689.

Mujuni P.F., Mgongo F.O.K., Kanuya N.L.: Ovarian cysts, a postpartum ovarian disorder affecting dairy cows in a tropical area. Anim. Reprod. Sci. 1993, 31: 175-186. 
Noble K.M., Tebble J.E., Harvey D., Dobson H.: Ultrasonography and hormone profiles of persistent ovarian follicles (cysts) induced with low doses of progesterone in cattle. J. Reprod. Fertil. 2000, 120: 361-366.

Peukert-Adam I., Schwarz R., Grunert E.: Zur Follikel-Lutein-Zyste des Rindes. Morphologie und Diskussion ihrer Bedeutung als Sterilitätsfaktor. Zbl. Vet. Med. A 1983, 30, 410-428.

Prakash B. S., Meyer H. H. D., Schallenberger E., van de Wiel D. F. M.: Development of a sensitive enzymeimmunoassay (EIA) for progesterone determination in unextracted bovine plasma using the second antibody technique. J. Steroid. Biochem. 1987, 28: 623-627.

Ribadu A.Y., Ward W.R., Dobson H.: Comparative evaluation of ovarian structures in cattle by palpation per rectum, ultrasonography and plasma progesterone concentration. Vet. Rec. 1994, 135: 452-457.

Richards M.W., Wettemann R.P., Schoenemann H.M.: Nutritional anestrus in beef cows: Body weight change, body condition, luteinizing hormone in serum and ovarian activity. J. Anim. Sci. 1989, 67: 1520-1526.

Schopper D., Claus R.: Progesteron concentrations in milkfat around ovulation in the dairy cow: Differences between observed and silent heat. Zuchthyg. 1986, 21: 237-240.

Silvia W.J., Hatler T.B. Nugent A.M. Laranja da Fonseca L.F.: Ovarian follicular cysts in dairy cows: An abnormality in folliculogenesis. Dom. Anim. Endocrinol. 2002, 23: 167-177.

Staufenbiel R.: Konditionsbeurteilung von Milchkühen mit Hilfe der sonographischen Rückenfettdickenmessung. Prakt. Tierarzt, coll. vet., 1997, XXVII: 87-92.

Studer E.: A veterinary perspective of on-farm evaluation of nutrition and reproduction. J. Dairy Sci. 1998, 81: 872-876.

Weber E.: Grundriss der biologischen Statistik. 10. Aufl., G. Fischer Verlag Stuttgart, 1995.

Wiltbank M.C., Gümen A., Sartori R.: Physiological classification of anovulatory conditions in cattle. Theriogenology 2002, 57:21-52.

\section{Korrespondenzadresse}

Martin Hasler, Blumenweg 11, CH-9113 Degersheim; Fax: 00417137122 75; E-mail: martin_hasler@bluewin.ch 


\section{Lebenslauf}

Name

Geburtsdatum

Geburtsort

Nationalität

Heimatort

$1974-1979$

$1980-1983$

$1989-1992$

1992

$1992-1998$

1998

$1999-2000$

Seit 2000

07.11 .2003
Martin Ludwig, Hasler

19.05.1967

Vaduz

Fürstentum Liechtenstein

Eschen

Primarschule in Vaduz

Sekundarschule in Vaduz

AKAD-Zweitwegmatura

Matura Typus B

Studium der Veterinärmedizin an der Veterinärmedizinischen

Fakultät der Universität Zürich, Schweiz

Abschlussprüfung an der Veterinärmedizinischen Fakultät der Universität Zürich, Schweiz

Assistentenstelle in der Tierarztpraxis Stockrüti, CH- 8572 Berg

Assistentenstelle in der Tierarztpraxis Dr. U. Eigenmann, CH- 9113 Degersheim 


